\title{
Coupled System of Boundary Value Problems by Galerkin Method with Cubic B-Splines
}

\author{
Kasi Viswanadham K.N.S. \\ Department of Mathematics, National Institute of Technology, Warangal - 506 004, India
}

\begin{abstract}
Coupled system of second order linear and nonlinear boundary value problems occur in various fields of Science and Engineering including heat and mass transfer. In the formulation of the problem, any one of 81 possible types of boundary conditions may occur. These 81 possible boundary conditions are written as a combination of four boundary conditions. To solve a coupled system of boundary value problem with these converted boundary conditions, a Galerkin method with cubic Bsplines as basis functions has been developed. The basis functions have been redefined into a new set of basis functions which vanish on the boundary. The nonlinear boundary value problems are solved with the help of quasilinearization technique. Several linear and nonlinear boundary value problems are presented to test the efficiency of the proposed method and found that numerical results obtained by the present method are in good agreement with the exact solutions available in the literature.
\end{abstract}

\section{Introduction}

It is well known that, many problems in the areas of science and engineering are modeled by second order ordinary differential systems [1-3]. There are only few methods available to solve the coupled system of linear or nonlinear differential equations. In this paper we considered a system of second order linear boundary value problems of the type

$$
\begin{array}{ll}
a_{0}(x) u_{1}^{\prime \prime}+a_{1}(x) u_{1}^{\prime}+a_{2}(x) u_{1} & a<x<b \\
+a_{3}(x) u_{2}^{\prime \prime}+a_{4}(x) u_{2}^{\prime}+a_{5}(x) u_{2}=f_{1}(x) & \\
b_{0}(x) u_{1}^{\prime \prime}+b_{1}(x) u_{1}^{\prime}+b_{2}(x) u_{1} & a<x<b \\
+b_{3}(x) u_{2}^{\prime \prime}+b_{4}(x) u_{2}^{\prime}+b_{5}(x) u_{2}=f_{2}(x) &
\end{array}
$$

subject to the boundary conditions

$$
\begin{aligned}
& \sigma_{1} u_{1}^{\prime}(a)+\sigma_{2} u_{1}(a)=u_{10} \\
& \sigma_{3} u_{1}^{\prime}(b)+\sigma_{4} u_{1}(b)=u_{11} \\
& \mu_{1} u_{2}^{\prime}(a)+\mu_{2} u_{2}(a)=u_{20} \\
& \mu_{3} u_{2}^{\prime}(b)+\mu_{4} u_{2}(b)=u_{21}
\end{aligned}
$$

where $\sigma_{1}, \sigma_{2}, \sigma_{3}, \sigma_{4}, \mu_{1}, \mu_{2}, \mu_{3}, \mu_{4}, u_{10}, u_{11}, u_{20}, u_{21}$ are finite real constants and $a_{0}(x), a_{1}(x), \ldots, a_{5}(x), b_{0}(x), b_{1}(x)$, $\ldots, b_{5}(x), f_{1}(x), f_{2}(x)$ are all continuous on $[a, b]$.
In many of applications we get a system of second order boundary value problems with both linear and nonlinear type. Here we considered a linear system of second order boundary value problems (1.1)-(1.2) along with 81 possible types of boundary conditions. If there is a coupled nonlinear system of second order boundary value problems, it can be converted into a sequence of coupled system of linear second order boundary value problems by using quasilinearization technique [4]. The limit of solutions of these generated linear boundary value problems is the solution of the nonlinear boundary value problem. Geng \& Cui [5] and Dehghan \& Abbas [6] solved coupled linear and coupled nonlinear system of boundary value problems with homogeneous boundary conditions only. In [6] they presented a sinc collocation method to solve the system. Several authors presented different numerical techniques of solving coupled system of second order boundary value problems [7-9].

In the next section we present the definition of cubic B-splines. In finite element method, the approximate solution can be written as a linear combination of basis functions which constitute a basis for the approximation space under consideration. The finite element method viz., Galerkin method produces a weak form of approximate solution for a given differential equation and is unique under appropriate conditions [10,11] irrespective of properties of a given differential operator and weak solution is also a classical solution of given differential equation provided sufficient attention is given to the boundary conditions[12]. The attention to boundary conditions is presented in section 3 . In section 
4 the Galerkin method to solve the given coupled linear system (1) has been presented. In section 5 the solution procedure to find the nodal parameters has been presented. In section 6 the proposed method is tested on two coupled linear systems and one coupled non linear system. The solution of coupled nonlinear system has been obtained as the limit of sequence of coupled linear problems generated by quasilinearization technique [4]. Finally, we presented the conclusions in the last section.

\section{Definition of Cubic B-Splines}

The existence of cubic B-spline interpolate $s(x)$ to a function $f(x)$ in a closed interval $[a, b]$ for spaced knots

$$
a=x_{0}<x_{1}<\ldots<x_{n-1}<x_{n}=b
$$

is established by constructing it. The construction of $s(x)$ is done with the help of cubic B-splines. Introduce six additional knots $x_{-3}, x_{-2}, x_{-1}, x_{n+1}, x_{n+2}, x_{n+3}$ such that $x_{-3}<x_{-2}<x_{-1}<x_{0}$ and $x_{n}<x_{n+1}<x_{n+2}<x_{n+3}$.

Now the cubic B- splines $B_{i}(x)$, given in $[13,14]$ are defined by

$$
B_{i}(x)=\left\{\begin{array}{lc}
\sum_{r=i-2}^{i+2} \frac{\left(x_{r}-x\right)_{+}^{3}}{\pi^{\prime}\left(x_{r}\right)} & \text { if } x \in\left[x_{i-2}, x_{i+2}\right] \\
0 & \text { otherwise }
\end{array}\right.
$$

where

$$
\left(x_{r}-x\right)_{+}^{3}=\left\{\begin{array}{cl}
\left(x_{r}-x\right)^{3} & \text { if } x_{r} \geq x \\
0 & \text { if } x_{r} \leq x
\end{array}\right.
$$

and

$$
\pi(x)=\left(x-x_{i-2}\right)\left(x-x_{i-1}\right)\left(x-x_{i}\right)\left(x-x_{i+1}\right)\left(x-x_{i+2}\right) .
$$

It can be shown that the set $\left\{B_{-1}(x), B_{0}(x), B_{1}(x), \ldots\right.$, $\left.B_{n}(x), B_{n+1}(x)\right\}$ forms a basis for the space $S_{3}(\pi)$ of cubic polynomial splines [15]. Schoenberg [16] has proved that the cubic B-splines are the unique non zero splines of smallest compact support with knots at

$x_{-3}<x_{-2}<x_{-1}<x_{0}<x_{1}<\ldots<x_{n}<x_{n+1}<x_{n+2}<x_{n+3}$.

\section{Attention to Boundary Conditions}

To solve the coupled linear system of boundary value problem (1) by Galerkin method with cubic B-splines as basis functions, we approximate the solutions $u_{1}$ and $u_{2}$ as

$$
\begin{aligned}
& u_{1}(x)=\sum_{j=-1}^{n+1} \alpha_{j} B_{j}(x) \\
& u_{2}(x)=\sum_{j=-1}^{n+1} \beta_{j} B_{j}(x)
\end{aligned}
$$

where $\alpha_{j}$ 's and $\beta_{j}$ 's are nodal parameters to be determined and $B_{j}(x)$ 's are cubic B-spline basis functions.

When the chosen approximation satisfies the prescribed boundary conditions, it gives better approximation results. In view of this, the basis functions are redefined into a new set of basis functions which vanish on the boundary along with the nonhomogeneous part which takes care of the prescribed boundary conditions. In the set of cubic B-splines $\left\{B_{-1}(x), B_{0}(x)\right.$, $\left.B_{1}(x), \ldots, B_{n-1}(x), B_{n}(x), B_{n+1}(x)\right\}$, the basis functions $B_{-1}(x), \quad B_{0}(x), B_{1}(x), \quad B_{\mathrm{n}-1}(x), B_{\mathrm{n}}(x)$ and $B_{\mathrm{n}+1}(x)$ do not vanish at one of the boundary points. So, there is a necessity of redefining the basis functions into a new set of basis functions which vanish on the boundary. The procedure for redefining of the basis functions is as follows.

Applying the boundary conditions (1.3) for $u_{1}(x)$ and $u_{2}(x)$ defined in (2) and from the definition of cubic Bsplines described in section 2 , we get

$$
\begin{aligned}
& u_{10}=\sigma_{1} u_{1}^{\prime}(a)+\sigma_{2} u_{1}(a)=\sigma_{1} u_{1}^{\prime}\left(x_{0}\right)+\sigma_{2} u_{1}\left(x_{0}\right) \\
& =\sigma_{1}\left[\alpha_{-1} B_{-1}^{\prime}\left(x_{0}\right)+\alpha_{0} B_{0}^{\prime}\left(x_{0}\right)+\alpha_{1} B_{1}^{\prime}\left(x_{0}\right)\right] \\
& +\sigma_{2}\left[\alpha_{-1} B_{-1}\left(x_{0}\right)+\alpha_{0} B_{0}\left(x_{0}\right)+\alpha_{1} B_{1}\left(x_{0}\right)\right] \\
& u_{11}=\sigma_{3} u_{1}^{\prime}(b)+\sigma_{4} u_{1}(b)=\sigma_{3} u_{1}^{\prime}\left(x_{n}\right)+\sigma_{4} u_{1}\left(x_{n}\right) \\
& =\sigma_{3}\left[\alpha_{n-1} B_{n-1}^{\prime}\left(x_{n}\right)+\alpha_{n} B_{n}^{\prime}\left(x_{n}\right)+\alpha_{n+1} B_{n+1}^{\prime}\left(x_{n}\right)\right] \\
& +\sigma_{4}\left[\alpha_{n-1} B_{n-1}\left(x_{n}\right)+\alpha_{n} B_{n}\left(x_{n}\right)+\alpha_{n+1} B_{n+1}\left(x_{n}\right)\right] \\
& u_{20}=\mu_{1} u_{2}^{\prime}(a)+\mu_{2} u_{2}(a)=\mu_{1} u_{2}^{\prime}\left(x_{0}\right)+\mu_{2} u_{2}\left(x_{0}\right) \\
& =\mu_{1}\left[\beta_{-1} B_{-1}^{\prime}\left(x_{0}\right)+\beta_{0} B_{0}^{\prime}\left(x_{0}\right)+\beta_{1} B_{1}^{\prime}\left(x_{0}\right)\right] \\
& +\mu_{2}\left[\beta_{-1} B_{-1}\left(x_{0}\right)+\beta_{0} B_{0}\left(x_{0}\right)+\beta_{1} B_{1}\left(x_{1}\right)\right] \\
& u_{21}=\mu_{3} u_{2}^{\prime}(b)+\mu_{4} u_{2}(b)=\mu_{3} u_{2}^{\prime}\left(x_{n}\right)+\mu_{4} u_{2}\left(x_{n}\right) \\
& =\mu_{3}\left[\beta_{n-1} B_{n-1}^{\prime}\left(x_{n}\right)+\beta_{n} B_{n}^{\prime}\left(x_{n}\right)+\beta_{n+1} B_{n+1}{ }^{\prime}\left(x_{n}\right)\right] \\
& +\mu_{4}\left[\beta_{n-1} B_{n-1}\left(x_{n}\right)+\beta_{n} B_{n}\left(x_{n}\right)+\beta_{n+1} B_{n+1}\left(x_{n}\right)\right]
\end{aligned}
$$

Now eliminating $\alpha_{-1}, \alpha_{n+1}, \beta_{-1}$ and $\beta_{n+1}$ from the equations (3.1) to (3.6), we get

$$
\begin{aligned}
& u_{1}(x)=w_{1}(x)+\sum_{j=0}^{n} \alpha_{j} \tilde{B}_{j}(x) \\
& u_{2}(x)=w_{2}(x)+\sum_{j=0}^{n} \beta_{j} \hat{B}_{j}(x)
\end{aligned}
$$

where

$$
\begin{gathered}
w_{1}(x)=\frac{u_{10}}{\sigma_{1} B_{-1}^{\prime}\left(x_{0}\right)+\sigma_{2} B_{-1}\left(x_{0}\right)} B_{-1}(x) \\
+\frac{u_{11}}{\sigma_{3} B_{n+1}{ }^{\prime}\left(x_{n}\right)+\sigma_{4} B_{n+1}\left(x_{n}\right)} B_{n+1}(x) \\
w_{2}(x)=\frac{u_{20}}{\mu_{1} B_{-1}^{\prime}\left(x_{0}\right)+\mu_{2} B_{-1}\left(x_{0}\right)} B_{-1}(x) \\
+\frac{u_{21}}{\mu_{3} B_{n+1}{ }^{\prime}\left(x_{n}\right)+\mu_{4} B_{n+1}\left(x_{n}\right)} B_{n+1}(x) \\
\tilde{B}_{j}(x)=\left\{\begin{array}{c}
B_{j}(x)-\frac{\sigma_{1} B_{j}^{\prime}\left(x_{0}\right)+\sigma_{2} B_{j}\left(x_{0}\right)}{\sigma_{1} B_{-1}^{\prime}\left(x_{0}\right)+\sigma_{2} B_{-1}\left(x_{0}\right)} B_{-1}(x) \quad \text { for } \quad j=0,1 \\
B_{j}(x) \text { for } j=2,3, \ldots, n-2 \\
B_{j}(x)-\frac{\sigma_{3} B_{j}^{\prime}\left(x_{n}\right)+\sigma_{4} B_{j}\left(x_{n}\right)}{\sigma_{3} B_{n+1}^{\prime}\left(x_{n}\right)+\sigma_{4} B_{n+1}\left(x_{n}\right)} B_{n+1}(x) \quad \text { for } \quad j=n-1, n
\end{array}\right.
\end{gathered}
$$




$$
\hat{B}_{j}(x)=\left\{\begin{array}{c}
B_{j}(x)-\frac{\mu_{1} B_{j}^{\prime}\left(x_{0}\right)+\mu_{2} B_{j}\left(x_{0}\right)}{\mu_{1} B_{-1}^{\prime}\left(x_{0}\right)+\mu_{2} B_{-1}\left(x_{0}\right)} B_{-1}(x) \text { for } \quad j=0,1 \\
B_{j}(x) \text { for } \quad j=2,3, \ldots, n-2 \\
B_{j}(x)-\frac{\mu_{3} B_{j}^{\prime}\left(x_{n}\right)+\mu_{4} B_{j}\left(x_{n}\right)}{\mu_{3} B_{n+1}^{\prime}\left(x_{n}\right)+\mu_{4} B_{n+1}\left(x_{n}\right)} B_{n+1}(x) \quad \text { for } j=n-1, n
\end{array}\right.
$$

The new set of basis functions for the approximations $u_{1}(x)$ and $u_{2}(x)$ are $\left\{\tilde{B}_{j}(x), j=0,1, \ldots, n\right\}$ and $\left\{\hat{B}_{j}(x), j=0,1, \ldots, n\right\}$ respectively. Here $w_{l}(x)$ and $w_{2}(x)$ take care of given set of boundary conditions and $\tilde{B}_{j}(x)$ 's, $\hat{B}_{j}(x)$ 's vanish on the boundary.

\section{Description of the method}

Applying the Galerkin method with the redefined set of basis functions $\tilde{B}_{i}(x)$ and $\hat{B}_{i}(x), i=0,1, \ldots, n-1, n$ to the problem (1), we get

$$
\begin{aligned}
& \int_{x_{0}}^{x_{n}}\left[a_{0}(x) u_{1}^{\prime \prime}+a_{1}(x) u_{1}^{\prime}+a_{2}(x) u_{1}\right] \tilde{B}_{i}(x) d x \\
+ & \int_{x_{0}}^{x_{n}}\left[a_{3}(x) u_{2}^{\prime \prime}+a_{4}(x) u_{2}^{\prime}+a_{5}(x) u_{2}\right] \tilde{B}_{i}(x) d x \\
= & \int_{x_{0}}^{x_{n}} f_{1}(x) \tilde{B}_{i}(x) d x, \quad \text { for } i=0,1, \ldots, n \\
& \int_{x_{0}}^{x_{n}}\left[b_{0}(x) u_{1}^{\prime \prime}+b_{1}(x) u_{1}^{\prime}+b_{2}(x) u_{1}\right] \hat{B}_{i}(x) d x \\
+ & \int_{x_{0}}^{x_{n}}\left[b_{3}(x) u_{2}^{\prime \prime}+b_{4}(x) u_{2}^{\prime}+b_{5}(x) u_{2}\right] \hat{B}_{i}(x) d x \\
= & \int_{x_{0}}^{x_{n}} f_{2}(x) \hat{B}_{i}(x) d x, \quad \text { for } i=0,1, \ldots, n
\end{aligned}
$$

Substitute the approximations for $u_{1}(x)$ and $u_{2}(x)$ given in (3.7) and (3.8) in (4.1) and (4.2), and after rearranging the terms for resulting equations, we get a coupled system of equations in the matrix form as

where

$$
\begin{aligned}
& A \alpha+B \beta=F_{1} \\
& C \alpha+D \beta=F_{2}
\end{aligned}
$$

$$
\mathbf{A}=\left[a_{i j}\right]
$$

$$
\begin{gathered}
a_{i j}=\int_{x_{0}}^{x_{n}}\left\{a_{0}(x) \frac{d^{2}}{d x^{2}}\left[\tilde{B}_{j}(x)\right]+a_{1}(x) \frac{d}{d x}\left[\tilde{B}_{j}(x)\right]\right. \\
\left.+a_{2}(x) \tilde{B}_{j}(x)\right\} \tilde{B}_{i}(x) d x \\
\text { for } i=0,1,2, \ldots, n, j=0,1,2, \ldots n \\
\mathbf{B}=\left[b_{i j}\right] ;
\end{gathered}
$$

$$
\begin{aligned}
& b_{i j}=\int_{x_{0}}^{x_{n}}\left\{a_{3}(x) \frac{d^{2}}{d x^{2}}\left[\hat{B}_{j}(x)\right]+a_{4}(x) \frac{d}{d x}\left[\hat{B}_{j}(x)\right]\right. \\
& \left.+a_{5}(x) \hat{B}_{j}(x)\right\} \tilde{B}_{i}(x) d x \\
& \text { for } i=0,1,2, \ldots, n, j=0,1,2, \ldots n \\
& \mathbf{C}=\left[c_{i j}\right] \text {; } \\
& c_{i j}=\int_{x_{0}}^{x_{n}}\left\{b_{0}(x) \frac{d^{2}}{d x^{2}}\left[\tilde{B}_{j}(x)\right]+b_{1}(x) \frac{d}{d x}\left[\tilde{B}_{j}(x)\right]\right. \\
& \left.+b_{2}(x) \tilde{B}_{j}(x)\right\} \hat{B}_{i}(x) d x \\
& \text { for } i=0,1,2, \ldots, n, j=0,1,2, \ldots n \\
& \mathbf{D}=\left[d_{i j}\right] \\
& d_{i j}=\int_{x_{0}}^{x_{n}}\left\{b_{3}(x) \frac{d^{2}}{d x^{2}}\left[\hat{B}_{j}(x)\right]+b_{4}(x) \frac{d}{d x}\left[\hat{B}_{j}(x)\right]\right. \\
& \left.+b_{5}(x) \hat{B}_{j}(x)\right\} \hat{B}_{i}(x) d x \\
& \text { for } i=0,1,2, \ldots, n, j=0,1,2, \ldots n \\
& \mathbf{F}_{\mathbf{1}}=\left[f_{1 i}\right] \\
& f_{1 i}=\int_{x_{0}}^{x_{n}}\left[f_{1}(x)-\left\{a_{0}(x) \frac{d^{2} w_{1}}{d x^{2}}+a_{1}(x) \frac{d w_{1}}{d x}+a_{2}(x) w_{1}(x)\right.\right. \\
& \left.\left.+a_{3}(x) \frac{d^{2} w_{2}}{d x^{2}}+a_{4}(x) \frac{d w_{2}}{d x}+a_{5}(x) w_{2}(x)\right\}\right] \tilde{B}_{i}(x) d x \\
& \mathbf{F}_{2}=\left[f_{2 i}\right] \text {; } \\
& f_{2 i}=\int_{x_{0}}^{x_{n}}\left[f_{2}(x)-\left\{b_{0}(x) \frac{d^{2} w_{1}}{d x^{2}}+b_{1}(x) \frac{d w_{1}}{d x}+b_{2}(x) w_{1}(x)\right.\right. \\
& \left.\left.+b_{3}(x) \frac{d^{2} w_{2}}{d x^{2}}+b_{4}(x) \frac{d w_{2}}{d x}+b_{5}(x) w_{2}(x)\right\}\right] \hat{B}_{i}(x) d x
\end{aligned}
$$

and

$$
\boldsymbol{\alpha}=\left[\begin{array}{llll}
\alpha_{0} & \alpha_{1} & \cdots & \alpha_{n}
\end{array}\right]^{T}, \quad \boldsymbol{\beta}=\left[\begin{array}{llll}
\beta_{0} & \beta_{1} & \cdots & \beta_{n}
\end{array}\right]^{T}
$$

\section{Solution procedure to find the nodal parameters}

A typical integral element in any of the matrices $\mathbf{A}, \mathbf{B}, \mathbf{C}$ and $\mathbf{D}$ is $\sum_{m=0}^{n-1} \mathrm{I}_{m}$,

where $\mathrm{I}_{m}=\int_{x_{m}}^{x_{m+1}} \mathrm{r}_{i}(x) \mathrm{r}_{j}(x) \mathrm{Z}(x) d x$ and $\mathrm{r}_{i}(x), \mathrm{r}_{j}(x)$ are the basis functions or their derivatives. It may be noted that $\mathrm{I}_{m}=0$ if $\left(x_{i-2}, x_{i+2}\right) \cap\left(x_{j-2}, x_{j+2}\right) \cap\left(x_{m}, x_{m+1}\right)=\phi$. To evaluate each $\mathrm{I}_{m}$, we employed 4-point Gauss-Legendre quadrature formula. Thus the matrices A, B, C and D described in (4.5) to (4.8) are seven diagonal band matrices. We solve the coupled system of equations (4.3)-(4.4) by using the following iteration formula.

$$
\begin{aligned}
& \mathbf{A} \alpha^{(r+1)}=\mathbf{F}_{1}-\mathbf{B} \beta^{(r)} \\
& \mathbf{D} \beta^{(r+1)}=\mathbf{F}_{\mathbf{2}}-\mathbf{C} \alpha^{(r+1)}, \quad r=0,1,2, \ldots
\end{aligned}
$$


The nodal parameters $\alpha_{i}$ 's and $\beta_{i}$ 's can be obtained from the above system by using the band matrix solution package. We have used the FORTRAN-90 program to solve the boundary value problems (1) by the proposed method.

\section{Numerical Examples}

To test the applicability of the proposed method, we considered two examples of coupled linear systems and one coupled non linear system. The numerical results for these examples are compared with the results available in the literature.

\section{Example 1:}

Consider the following coupled linear system of boundary value problem

$$
\begin{gathered}
u^{\prime \prime}(x)+u^{\prime}(x)+x u(x)+v^{\prime}(x)+2 x v(x)=f_{1}(x), \\
0 \leq x \leq 1, \\
2 u^{\prime}(x)+x^{2} u(x)+v^{\prime \prime}(x)+v(x)=f_{2}(x) \\
0 \leq x \leq 1,
\end{gathered}
$$

subject to $u(0)=u(1)=0, v(0)=v(1)=0$ where

$$
\begin{aligned}
f_{1}(x)= & -2(1+x) \cos x+\pi \cos \pi x+2 x \sin \pi x \\
& +\left(4 x-2 x^{2}-4\right) \sin x \\
f_{2}(x)= & 4(1-x) \cos x+2\left(-2+x^{2}-x^{3}\right) \sin x \\
& +\left(1-\pi^{2}\right) \sin \pi x
\end{aligned}
$$

The exact solutions of $u$ and $v$ are $u(x)=2(1-x) \sin (x)$ and $v(x)=\sin (\pi x)$. The proposed method is tested on the problem (5.1) to (5.3). Numerical results obtained by the proposed method are presented in table 1 and table 2 and compared with the results in [5] and [6]. The maximum absolute error obtained by the proposed method is compared with that of obtained in [5] and [6] in table 3.

Table 1

Numerical results for the variable $u$ in Example 1

\begin{tabular}{|c|c|c|c|}
\hline$x$ & $\begin{array}{c}\text { Absolute } \\
\text { error by } \\
\text { proposed } \\
\text { method } \\
\text { with 10 } \\
\text { intervals }\end{array}$ & $\begin{array}{c}\text { Absolute } \\
\text { error by the } \\
\text { method [5] } \\
\text { with 32 } \\
\text { intervals }\end{array}$ & $\begin{array}{c}\text { Absolute } \\
\text { error by the } \\
\text { method [6] } \\
\text { with 32 } \\
\text { intervals }\end{array}$ \\
\hline 0.08 & $8.18 \times 10^{-6}$ & $3.3 \times 10^{-3}$ & $3.2 \times 10^{-3}$ \\
\hline 0.24 & $1.65 \times 10^{-5}$ & $7.7 \times 10^{-3}$ & $9.2 \times 10^{-4}$ \\
\hline 0.40 & $1.66 \times 10^{-5}$ & $9.7 \times 10^{-3}$ & $2.0 \times 10^{-3}$ \\
\hline 0.56 & $1.20 \times 10^{-5}$ & $9.5 \times 10^{-3}$ & $2.2 \times 10^{-4}$ \\
\hline 0.72 & $5.71 \times 10^{-5}$ & $7.3 \times 10^{-3}$ & $4.1 \times 10^{-3}$ \\
\hline 0.88 & $9.65 \times 10^{-6}$ & $3.4 \times 10^{-3}$ & $1.0 \times 10^{-2}$ \\
\hline 0.96 & $1.41 \times 10^{-6}$ & $1.1 \times 10^{-3}$ & $1.1 \times 10^{-3}$ \\
\hline
\end{tabular}

Table 2

Numerical results for the variable $v$ in Example 1

\begin{tabular}{|c|c|c|c|}
\hline$x$ & $\begin{array}{c}\text { Absolute error } \\
\text { by proposed } \\
\text { method } \\
\text { with 10 } \\
\text { intervals }\end{array}$ & $\begin{array}{c}\text { Absolute } \\
\text { error by the } \\
\text { method [5] } \\
\text { with 32 } \\
\text { intervals }\end{array}$ & $\begin{array}{c}\text { Absolute } \\
\text { error by the } \\
\text { method [6] } \\
\text { with 32 } \\
\text { intervals }\end{array}$ \\
\hline 0.08 & $2.32 \times 10^{-5}$ & $7.7 \times 10^{-3}$ & $1.5 \times 10^{-3}$ \\
\hline 0.24 & $6.22 \times 10^{-5}$ & $2.0 \times 10^{-2}$ & $7.0 \times 10^{-3}$ \\
\hline 0.40 & $8.38 \times 10^{-5}$ & $2.7 \times 10^{-2}$ & $7.4 \times 10^{-3}$ \\
\hline 0.56 & $8.52 \times 10^{-5}$ & $2.7 \times 10^{-2}$ & $1.0 \times 10^{-2}$ \\
\hline 0.72 & $6.60 \times 10^{-5}$ & $2.0 \times 10^{-2}$ & $4.4 \times 10^{-3}$ \\
\hline 0.88 & $3.14 \times 10^{-5}$ & $9.4 \times 10^{-3}$ & $2.1 \times 10^{-2}$ \\
\hline 0.96 & $1.07 \times 10^{-5}$ & $3.1 \times 10^{-3}$ & $6.9 \times 10^{-3}$ \\
\hline
\end{tabular}

Table 3

Maximum Absolute Errors for the Example 1

\begin{tabular}{|c|c|c|c|}
\hline Variable & $\begin{array}{c}\text { By the } \\
\text { proposed } \\
\text { method } \\
\text { with 10 } \\
\text { intervals }\end{array}$ & $\begin{array}{c}\text { By the } \\
\text { method } \\
\text { in [5] } \\
\text { with 32 } \\
\text { intervals }\end{array}$ & $\begin{array}{c}\text { By the } \\
\text { method } \\
\text { in [6] } \\
\text { with 32 } \\
\text { intervals }\end{array}$ \\
\hline$u$ & $1.66 \times 10^{-5}$ & $9.7 \times 10^{-3}$ & $1.0 \times 10^{-2}$ \\
\hline$v$ & $8.52 \times 10^{-5}$ & $2.7 \times 10^{-2}$ & $2.1 \times 10^{-2}$ \\
\hline
\end{tabular}

\section{Example 2:}

Consider the coupled linear system of boundary value problem

$$
\begin{aligned}
& u^{\prime \prime}+x u+2 v^{\prime}=x^{3}+x^{2}+2+2 \cos x, 0 \leq x \leq 1, \\
& u+v^{\prime \prime}+2 v=x^{2}+x+\sin x
\end{aligned}
$$

subject to the boundary conditions

$$
\begin{aligned}
& u^{\prime}(0)+u(0)=1 \\
& v^{\prime}(1)+v(1)=\cos 1+\sin 1 \\
& u(1)=2, v^{\prime}(0)=1
\end{aligned}
$$

The exact solutions $u$ and $v$ for the system (5.4) and (5.5) are $u(x)=x^{2}+x$ and $v(x)=\sin x$. The proposed method is tested on the problem (5.1) to (5.3) where the domain $[0,1]$ is divided into 10 equal intervals. Numerical results obtained by the proposed method are shown along with the exact solutions are presented in table 4 .

\section{Table 4}

\begin{tabular}{|c|c|c|}
\hline$x$ & $\begin{array}{c}\text { Absolute error } \\
\text { for } u(x)\end{array}$ & $\begin{array}{c}\text { Absolute error } \\
\text { for } v(x)\end{array}$ \\
\hline 0.0 & $0.37784 \mathrm{E}-05$ & $0.17698 \mathrm{E}-05$ \\
\hline 0.1 & $0.34035 \mathrm{E}-05$ & $0.17363 \mathrm{E}-05$ \\
\hline 0.2 & $0.30409 \mathrm{E}-05$ & $0.16417 \mathrm{E}-05$ \\
\hline 0.3 & $0.26974 \mathrm{E}-05$ & $0.14988 \mathrm{E}-05$ \\
\hline 0.4 & $0.23735 \mathrm{E}-05$ & $0.13226 \mathrm{E}-05$ \\
\hline 0.5 & $0.20593 \mathrm{E}-05$ & $0.11247 \mathrm{E}-05$ \\
\hline 0.6 & $0.17440 \mathrm{E}-05$ & $0.92328 \mathrm{E}-06$ \\
\hline 0.7 & $0.14138 \mathrm{E}-05$ & $0.73612 \mathrm{E}-06$ \\
\hline 0.8 & $0.10335 \mathrm{E}-05$ & $0.57280 \mathrm{E}-06$ \\
\hline 0.9 & $0.57340 \mathrm{E}-06$ & $0.44882 \mathrm{E}-06$ \\
\hline 1.0 & $0.00000 \mathrm{E}+00$ & $0.37789 \mathrm{E}-06$ \\
\hline
\end{tabular}




\section{Example 3:}

Consider the following coupled nonlinear system of boundary value problem

$$
\begin{aligned}
& \qquad \begin{array}{r}
u^{\prime \prime}(x)+x u(x)+2 x v(x)+x u^{2}(x)=f_{1}(x), \\
0 \leq x \leq 1
\end{array} \\
& \begin{array}{r}
x^{2} u(x)+v^{\prime}(x)+v(x)+\sin (x) v^{2}(x)=f_{2}(x), \\
0 \leq x \leq 1
\end{array} \\
& \text { subject to } u(0)=u(1)=0, v(0)=v(1)=0 \\
& \text { where } f_{1}(x)=2 x \sin \pi x+x^{5}-2 x^{4}+x^{2}-2 \\
& \text { and }
\end{aligned}
$$$$
f_{2}(x)=x^{3}(1-x)+\sin \pi x(1+\sin x \sin \pi x)+\pi \cos \pi x
$$

The exact solutions $u$ and $v$ for the (5.6)-(5.8) are $u(x)=x-x^{2}$ and $v(x)=\sin (\pi x)$. By the quasilinearization technique [4] the problem (5.6)-(5.8) has been converted into a sequence of coupled linear problems as

$$
\begin{aligned}
& u_{n+1}^{\prime \prime}(x)+\left[x\left(1+2 u_{n}\right)\right] u_{n+1}(x)+2 x v_{n+1}(x) \\
& =f_{1}(x)+x u_{n}^{2}(x)
\end{aligned}
$$

$x^{2} u_{n+1}(x)+v_{n+1}^{\prime}(x)+\left[1+2 \sin (x) v_{n}(x)\right] v_{n+1}(x)$

$=f_{2}(x)+\sin (x) v_{n}^{2}(x)$

subject to $u_{n+1}(0)=u_{n+1}(1)=0, v_{n+1}(0)=v_{n+1}(1)=0$ for $n=0,1,2, \ldots$

Here $u_{n+1}$ and $v_{n+1}$ are the $(n+1)^{\text {th }}$ approximations for $u$ and $v$ respectively. The proposed method is tested for the above problem. Numerical results obtained by the proposed method are presented in table 5 and table 6 and compared with the results in [5] and [6]. The maximum absolute error obtained by the proposed method is compared with that of obtained in [5] and [6] in table 7.

\begin{tabular}{|c|c|c|c|}
\hline Variable & $\begin{array}{l}\text { By the } \\
\text { proposed } \\
\text { Method } \\
\text { with } 10 \\
\text { intervals }\end{array}$ & $\begin{array}{l}\text { By the } \\
\text { method } \\
\text { in [5] } \\
\text { with } 32 \\
\text { intervals }\end{array}$ & $\begin{array}{l}\text { By the } \\
\text { method } \\
\text { in [6] } \\
\text { with } 32 \\
\text { intervals }\end{array}$ \\
\hline$u$ & $1.89 \times 10^{-5}$ & $2.2 \times 10^{-3}$ & $1.4 \times 10^{-4}$ \\
\hline$v$ & $4.16 \times 10^{-5}$ & $8.2 \times 10^{-3}$ & $3.1 \times 10^{-3}$ \\
\hline
\end{tabular}

Table 5

Numerical results for the variable $u$ in Example 3

\begin{tabular}{|c|c|c|c|}
\hline$x$ & $\begin{array}{c}\text { Absolute } \\
\text { error by } \\
\text { proposed } \\
\text { method } \\
\text { with 10 } \\
\text { intervals }\end{array}$ & $\begin{array}{c}\text { Absolute } \\
\text { error by the } \\
\text { method [5] } \\
\text { with 32 } \\
\text { intervals }\end{array}$ & $\begin{array}{c}\text { Absolute } \\
\text { error by the } \\
\text { method [6] } \\
\text { with 32 } \\
\text { intervals }\end{array}$ \\
\hline 0.08 & $3.27 \times 10^{-6}$ & $5.0 \times 10^{-4}$ & $1.4 \times 10^{-4}$ \\
\hline 0.24 & $9.40 \times 10^{-6}$ & $1.4 \times 10^{-3}$ & $4.4 \times 10^{-5}$ \\
\hline 0.40 & $1.46 \times 10^{-5}$ & $2.1 \times 10^{-3}$ & $6.7 \times 10^{-5}$ \\
\hline 0.56 & $1.84 \times 10^{-5}$ & $2.2 \times 10^{-3}$ & $9.3 \times 10^{-5}$ \\
\hline 0.72 & $1.89 \times 10^{-5}$ & $1.8 \times 10^{-3}$ & $4.9 \times 10^{-5}$ \\
\hline 0.88 & $1.27 \times 10^{-5}$ & $9.0 \times 10^{-4}$ & $8.6 \times 10^{-5}$ \\
\hline 0.96 & $4.99 \times 10^{-6}$ & $3.0 \times 10^{-4}$ & $7.1 \times 10^{-5}$ \\
\hline
\end{tabular}

\section{Table 6}

Numerical results for the variable $v$ in Example 3

\begin{tabular}{|c|c|c|c|}
\hline$x$ & $\begin{array}{c}\text { Absolute } \\
\text { error by } \\
\text { proposed } \\
\text { method } \\
\text { with 10 } \\
\text { intervals }\end{array}$ & $\begin{array}{c}\text { Absolute } \\
\text { error by the } \\
\text { method [5] } \\
\text { with 32 } \\
\text { intervals }\end{array}$ & $\begin{array}{c}\text { Absolute } \\
\text { error by the } \\
\text { method [6] } \\
\text { with 32 } \\
\text { intervals }\end{array}$ \\
\hline 0.08 & $1.64 \times 10^{-5}$ & $2.0 \times 10^{-3}$ & $2.4 \times 10^{-4}$ \\
\hline 0.24 & $7.63 \times 10^{-6}$ & $5.6 \times 10^{-3}$ & $2.3 \times 10^{-3}$ \\
\hline 0.40 & $4.90 \times 10^{-5}$ & $7.9 \times 10^{-3}$ & $8.9 \times 10^{-4}$ \\
\hline 0.56 & $7.39 \times 10^{-6}$ & $8.2 \times 10^{-3}$ & $1.4 \times 10^{-3}$ \\
\hline 0.72 & $1.76 \times 10^{-5}$ & $6.5 \times 10^{-3}$ & $3.1 \times 10^{-3}$ \\
\hline 0.88 & $4.16 \times 10^{-5}$ & $3.1 \times 10^{-3}$ & $1.6 \times 10^{-3}$ \\
\hline 0.96 & $1.79 \times 10^{-5}$ & $1.0 \times 10^{-3}$ & $9.8 \times 10^{-4}$ \\
\hline
\end{tabular}

Table 7

Maximum Absolute Errors for the Example 3

\section{Conclusions}

In this paper, we have developed a Galerkin method with cubic B-splines as basis functions to solve a coupled system of linear second order boundary value problems. The cubic B-splines basis set have been redefined into a new set of basis functions which vanish on the boundary along with the nonhomogeneous part which takes care of the prescribed boundary conditions. The solution of a coupled nonlinear system has been obtained as the limit of sequence of coupled linear problems generated by quasilinearization technique. The proposed method is applied to solve two linear problems and one nonlinear problem to test the efficiency of the method. The numerical results obtained by the method are in good agreement with the exact solutions available in the literature. The maximum absolute errors obtained by the proposed method are less when compared with those of available in the literature. The objective of this paper is to present a simple technique to solve a coupled system of second order boundary value problems.

\section{References}

[1]. C Wafo soh, F.M. Mahomed, Internat. J. NonLinear Mech. 36, 671-677 (2001)

[2]. S Matthews, Parameter robust numerical methods for a system of two coupled singularly perturbed reaction diffusion equations, Master Thesis, School of Mathematical Sciences, Dublin city University, (2000)

[3]. K.N. Tomantschger, J. comput. Appl. Math. 140, 773-783 (2002) 
[4]. R.E Bellman and R.E. Kalaba, Quasilinearization and Nonlinear Boundary value problems, American Elsevier, New York (1965).

[5]. Fazhan Geng, Minggen Cui, J. Math. Anal. Appl. 327, 1167-1181 (2007)

[6]. Mehdi Dehgan, Abbas Saadatmandi, Math. Comput. Modelling 46, 1434-1441 (2007)

[7]. A. Tamilselvan, N. Ramanujan, V. Shanti, J. comput. Appl. Math. 202, 203-216 (2007)

[8]. H. B. Thompson, C. Tisdell, J. Math. Anal. Appl. 248, 333-347, (2000)

[9]. T. Valanarasu, N. Ramanujan, Appl. Math. Comput. 147(6), 227-240, (2004)

[10]. Lions J.L. and Magenes E, Non-Homogeneous Boundary Value Problems and Applications, Springer - Verlag, Berlin (1972).

[11]. Bers L.,John F. and Schecheter M., Partial Differential Equations, John Wiley Inter Science, New York, 1964.

[12]. Mitchell A.R. and Wait R., The Finite Element Method in Partial Differential Equations, John Wiley and Sons, London (1977).

[13]. M.G. Cox, Jour. Inst. Mathematics and Appl. 10, 134-149, (1972)

[14]. Carl de Boor, A practical guide to splines, Springer-Verlag (1978).

[15]. P. M. Prenter, Splines and Variational Methods, John-Wiley and Sons, New York, (1975)

[16]. I.J.Schoenberg, On Spline Functions, MRC Report 625, University of Wisconsin (1966). 